\title{
Correlation of TTF-1 immunoexpression and EGFR mutation spectrum in non-small cell lung carcinoma
}

\author{
Tripti Nakra ${ }^{1}$, Varsha Singh ${ }^{1 *}$, Aruna Nambirajan ${ }^{1 *}$, Prabhat Singh Malik², Anant Mohan ${ }^{3}$, Deepali Jain ${ }^{1}$ \\ 'Department of Pathology, All India Institute of Medical Sciences, New Delhi; \\ Department of Medical Oncology, Dr B.R. Ambedkar Institute Rotary Cancer Hospital, All India Institute of Medical Sciences, New Delhi; \\ ${ }^{3}$ Department of Pulmonary Medicine and Sleep Disorders, All India Institute of Medical Sciences, New Delhi, India
}

\begin{abstract}
Background: Thyroid transcription factor (TTF-1) is a diagnostic marker expressed in $75 \%-85 \%$ of primary lung adenocarcinomas (ACs). Activating mutations in the tyrosine kinase domain of the epidermal growth factor receptor (EGFR) gene is the most common targetable driver alteration in lung AC. Previous studies have shown a positive correlation between TTF-1 and EGFR mutation status. We aimed to determine the predictive value of TTF- 1 immunoexpression for underlying EGFR mutation status in a large Indian cohort. Methods: This retrospective designed study was conducted with medical record data from 2011 to 2020. All cases of primary lung AC and non-small cell lung carcinoma not otherwise specified (NSCLC, NOS) with known TTF-1 expression diagnosed by immunohistochemistry using 8G7G3/1 antibodies and EGFR mutation status diagnosed by quantitative polymerase chain reaction were retrieved, reviewed, and the results were analyzed. Results: Among 909 patient samples diagnosed as lung AC and NSCLC, NOS, TTF-1 was positive in 76.8\% cases (698/909) and EGFR mutations were detected in 29.6\% (269/909). A strong positive correlation was present between TTF-1 positivity and EGFR mutation status (odds ratio, $3.61 ; \mathrm{p}<.001$ ), with TTF-1 positivity showing high sensitivity $(90 \%)$ and negative predictive value (87\%) for EGFR mutation. TTF-1 immunoexpression did not show significant correlation with uncommon/dual EGFR mutations (odds ratio, 1.69; $p=.098$ ). EGFR-tyrosine kinase inhibitor therapy was significantly superior to chemotherapy among EGFR mutant cases irrespective of TTF-1 status; however, no significant differences among survival outcomes were observed. Conclusions: Our study confirms a strong positive correlation between TTF-1 expression and common EGFR mutations (exon 19 deletion and exon 21 L858R) in advanced lung AC with significantly high negative predictive value of TTF-1 for EGFR mutations.
\end{abstract}

Key Words: EGFR mutation; Lung adenocarcinoma; Non-small cell lung carcinoma; Thyroid transcription factor-1; Uncommon mutations

Received: January 28, 2021 Revised: April 2, 2021 Accepted: May 10, 2021

Corresponding Author: Deepali Jain, MD, DNB, Department of Pathology, All India Institute of Medical Sciences, New Delhi 110029, India

Tel: +91-9868895112, Fax: +91-1126549200, E-mail: deepalijain76@gmail.com

*Varsha Singh and Aruna Nambirajan contributed equally to this work.

Thyroid transcription factor 1 (TTF-1) is a regulatory transcription factor for maintaining peripheral lung function and is required for normal lung morphogenesis [1]. It is expressed normally in type II pneumocytes and non-ciliated bronchiolar epithelial cells, and this expression pattern is retained in 75\%$85 \%$ of primary lung adenocarcinomas (ACs) [2,3]. TTF-1 positivity is highly specific to lung $\mathrm{AC}$ among all non-neuroendocrine neoplasms of the lung and is used widely as an immunohistochemical (IHC) marker for diagnosis of AC of lung origin and in subtyping of non-small cell lung carcinoma (NSCLC) not otherwise specified (NOS) wherein TTF-1 positivity favors diagnosis of lung AC over that of squamous cell carcinoma [4].

$\mathrm{AC}$ is the most common histological subtype of lung carcino- ma, both worldwide and in India, with an increasing trend in incidence $[5,6]$. With discovery of mutations in the epidermal growth factor receptor $(E G F R)$ gene in up to one-third of all ACs and the high response rates of these patients to EGFR tyrosine kinase inhibitor (EGFR-TKI) therapy, the latter is the first-line treatment of choice in patients with advanced non-squamous NSCLC harboring EGFR mutations. In accordance, any lung carcinoma sample diagnosed as AC, or NSCLC, NOS on histology with or without IHC should be subjected to molecular testing (MT) for targetable alterations in EGFR, anaplastic lymphoma kinase ( $A L K$ ), or c-ros oncogene 1 (ROS1) genes at minimum [7].

One of the major constraints in diagnosis and clinical management of lung $\mathrm{AC}$ is the non-availability of adequate tumor 
tissue for MT. With nearly 70\% of all lung cancer patients presenting with advanced stage, small biopsies/cytology represent the major source of tumor tissue, and successful MT on such small samples requires triage of material by informed pathologists and trained technicians [8]. Further, in a developing country such as India, the majority of laboratories do not have facilities for MT, and the samples usually are deferred to a higher medical center, resulting in delay and difficulty in timely treatment decisions.

Unlike MT, IHC is a well-established technique practiced even in small laboratories, and IHC using the recommended TTF-1 $8 \mathrm{G} 7 \mathrm{G} 3 / 1$ mouse monoclonal antibody [9] is available in most laboratories. Limited studies have analyzed the correlation between TTF-1 expression and EGFR mutations in lung AC and have reported a positive correlation between the two. Further, like EGFR mutant ACs, TTF-1 positivity has been shown to be associated with longer overall survival (OS), especially in nonmetastatic NSCLC [10-12]. This retrospective study was undertaken to assess the correlation of TTF-1 IHC with EGFR mutation status to understand whether TTF-1 expression status can be used as a guide for prioritizing EGFR MT and/or for treatment decisions in resource-limited settings.

\section{MATERIALS AND METHODS}

\section{Case selection}

The current study was of a retrospective design spanning over duration of 9 years (January 2011 to February 2020), approved by the institutional ethics committee (IECPG-480/29.08.2016).

All primary lung $A C$ patients with available medical record data pertaining to both TTF-1 and EGFR mutation testing on surgical pathology samples were selected for this study. The present study cohort included patients from previously published data pertaining to EGFR mutation rate [13-15]. Histopathological slides were reviewed, and diagnosis was reconfirmed in accordance with WHO classification of tumours of the lung, pleura, thymus and heart (2015) [16]. Detailed histopathological examination and subtyping of all cases were performed. Clinicopathological data including age, sex, smoking status, specimen type, site of biopsy, IHC result of TTF-1, and EGFR mutation status were recorded from the histopathology requisition forms.

\section{Immunohistochemistry for TTF-1}

IHC for TTF-1 was performed on 4- $\mu$ m-thick, formalin-fixed, paraffin-embedded tissue sections as part of diagnostic work-up during routine clinical practice. Heat-induced epitope retrieval was conducted in citrate buffer at $\mathrm{pH}$ 6, followed by a 3-hour incubation period with TTF-1 antibody (1:400, mouse monoclonal antibody, clone 8G7G3/1, Bio-SB, Santa Barbara, CA, USA). A universal-labeled streptavidin biotin kit was used as a detection system. Nuclear staining of weak or strong intensity in $\geq 5 \%$ of tumor cells was considered positive for TTF- 1 expression [4]. For every case, negative and positive internal controls (normal lung tissue) or external controls (absence of normal lung parenchyma) were used.

\section{EGFR mutation analysis}

Real-time polymerase chain reaction-based EGFR mutation testing was conducted using the EGFR RGQ PCR Kit (Therascreen, Cat No. 870111, Qiagen, Hilden, Germany) to detect 29 hotspot mutations in EGFR exons 18-21.

\section{Statistical analysis}

Data was analyzed using GraphPad Prism ver. 8 (GraphPad Software Inc., San Diego, CA, USA). Categorical variables were tabulated as frequency and percentage. The chi-square or Fisher exact tests were used to analyze the categorical variables between TTF-1 expression and EGFR mutations. Patient age was represented in mean values with standard deviations. A $\mathrm{p}<.05$ was considered statistically significant.

\section{RESULTS}

A total of 909 patient samples were included in the study (Table 1). The mean age of the cohort was 57 years, with a male preponderance (male:female ratio of 2.4:1). Sample types were tumor resections ( $\mathrm{n}=19)$ and small biopsies $(\mathrm{n}=890)$, the latter also including lymph node excision biopsies and Tru-Cut biopsies from metastatic sites. TTF-1 was positive in $76.8 \%$ cases (698/909) (Fig. 1) and was significantly more prevalent among non-smokers $(\mathrm{p}<.001)$; however, there was no difference in prevalence among sex or stage-based groups. EGFR mutations were detected in $29.6 \%$ of cases (269/909). EGFR mutations were significantly more frequent among NSCLC occurring in females ( $46 \%$ vs. $23 \%$ in males, $\mathrm{p}=.001$ ), non-smokers ( $58 \%$ vs. $28 \%$ in smokers, $\mathrm{p}<.001)$, and in early stages of presentation $(67 \%$ vs. $41 \%$ in late stages, $\mathrm{p}=.011$ ). However, only a small proportion of our cases was diagnosed at earlier stages. The most common mutation detected was exon 19 deletion (146/269, 54.3\%), followed by exon 21 mutation encoding the L858R amino acid change (exon 21 L858R; 65/269, 24.2\%). Other less common mutations observed were in exon 21 L861Q (2/269, 0.7\%), exon 20 inser- 

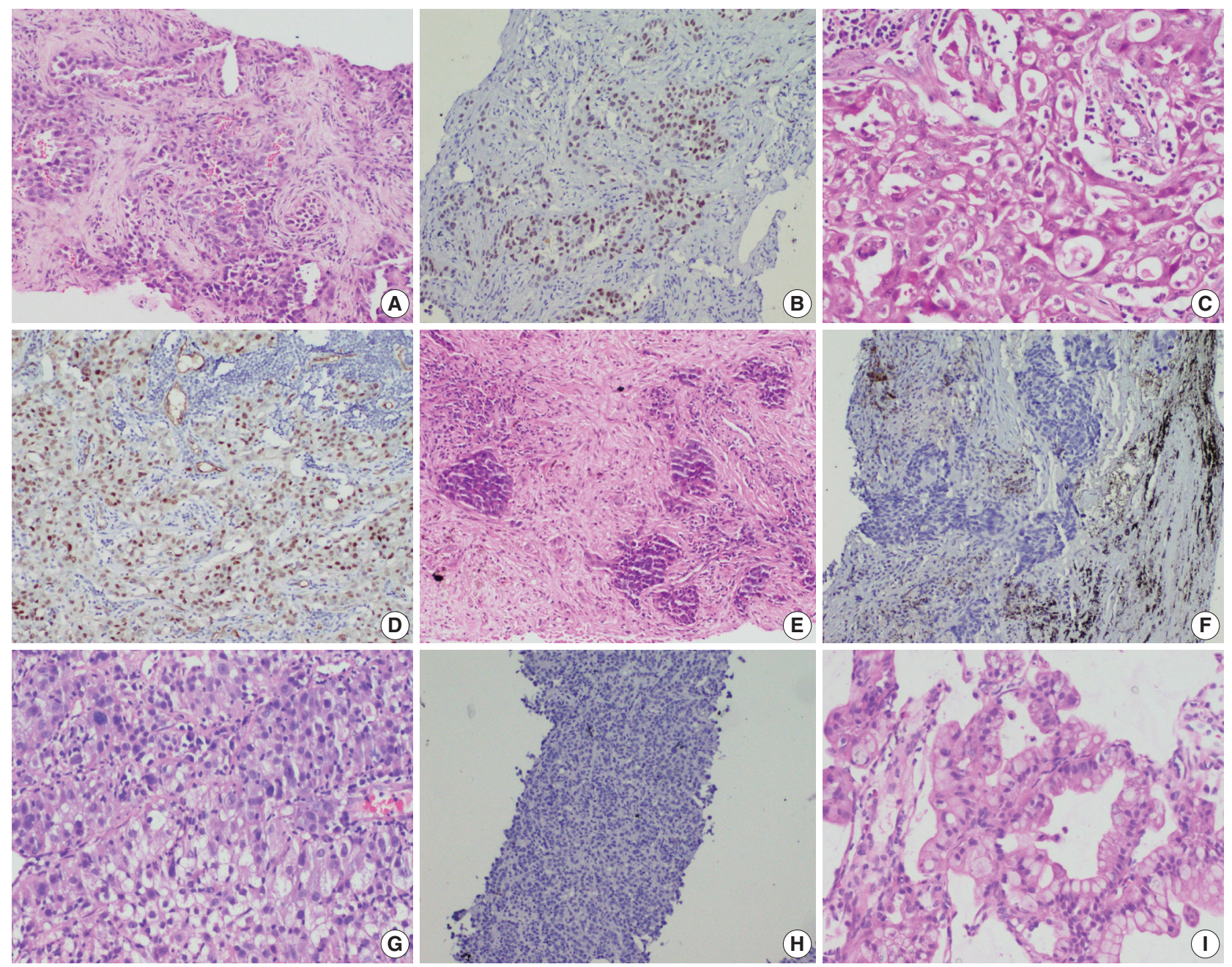

Fig. 1. Photomicrographs of non-small cell lung carcinoma (NSCLC). Epidermal growth factor receptor (EGFR) mutant lung adenocarcinoma with acinar and solid patterns (A) showing nuclear staining for thyroid transcription factor 1 (TTF-1) in tumor cells (B). EGFR wild type NSCLC with solid architecture (C) immunopositive for TTF-1 (D). Examples of EGFR mutant NSCLC not otherwise specified (NOS) (E) and EGFR wild type NSCLC, NOS $(G)$ that are negative for TTF-1 (F, H); EGFR mutant invasive mucinous adenocarcinoma (I).

tion (4/269, 1.5\%), exon 20 T790M (7/269, 2.6\%), exon 20 S768I (3/269, 1.1\%), and exon 18 G719X (3/269, 1.1\%). Dual mutations involving multiple exons were detected in 39/269 cases $(14.5 \%)$, the majority of which encoded the T790M amino acid change $(17 / 39,43.6 \%)$ in combination with other sensitive mutations. The rate of TTF-1 expression was lower among uncommon/dual mutations $(84 \%, 49 / 58)$ compared to exon 19 deletion $(91 \%, 133 / 146)$ and exon 21 L858R (92\%, 60/65), although this did not reach statistical significance (Table 1).

\section{Correlation between TTF-1 immunopositivity and EGFR mutation}

Ninety percent of all EGFR mutant NSCLCs were TTF-1 positive, with TTF-1 positivity showing high sensitivity (90\%) and negative predictive value (NPV; 87\%) for EGFR mutations. However, only $30 \%$ of all TTF-1-positive ACs harbored EGFR mutations, showing low specificity (29\%) and poor positive predictive value (PPV; 35\%) (Table 2). Considering that the prevalence of $E G F R$ mutations varies among clinicopathological subsets, in particular with sex and smoking status, we analyzed the correlation between TTF-1 and EGFR mutations in these subgroups separately. As expected, the PPV of TTF-1 positivity for EGFR mutations was highest among females (51\%) and nonsmokers (63\%), which are the two clinical groups that show a higher prevalence of $E G F R$ mutations. The lowest prevalence of EGFR mutations was among males (28\%) and smokers (33\%). Among individual types of mutations, PPV was higher with 
Table 1. Clinicopathological features of patients included in the study

\begin{tabular}{|c|c|c|c|c|c|c|c|}
\hline \multirow{2}{*}{ Parameter } & \multirow{2}{*}{ №. (\%) } & \multicolumn{3}{|c|}{ TTF-1 immunohistochemistry } & \multicolumn{3}{|c|}{ EGFR mutation status } \\
\hline & & Positive & Negative & $p$-value & Mutant & Wild type & p-value \\
\hline Total No. of patients & 909 & $698(76.8)$ & $211(23.2)$ & - & $269(29.6)$ & $640(70.4)$ & - \\
\hline Mean age at presentation (SD) & $57(12.3)$ & $57(12.4)$ & $57(12.3)$ & $>.05$ & $55(12.2)$ & $57(12.4)$ & $>.05$ \\
\hline Sex & & & & & & & .001 \\
\hline Male & $644(70.8)$ & $486(75.5)$ & $158(24.5)$ & .082 & $148(23.0)$ & $496(77.0)$ & \\
\hline Female & $265(29.2)$ & $212(80.0)$ & $53(20.0)$ & & $121(45.7)$ & $144(54.3)$ & \\
\hline Smoking status $(n=362)$ & & & & & & & $<.001$ \\
\hline Smoker & $183(50.6)$ & $122(66.7)$ & $61(33.3)$ & $<.001$ & $52(28.4)$ & $131(71.6)$ & \\
\hline Non-smoker & $179(49.4)$ & $155(86.6)$ & $24(13.4)$ & & $103(57.5)$ & $76(42.5)$ & \\
\hline Stage at presentation $(n=358)$ & & & & & & & .011 \\
\hline I-IIIA & $30(8.4)$ & $23(76.7)$ & 7 (23.3) & .594 & $20(66.7)$ & $10(33.3)$ & \\
\hline IIIB/IV & $328(91.6)$ & $251(76.5)$ & $77(23.5)$ & & $135(41.2)$ & $193(58.8)$ & \\
\hline First-line treatment $(n=383)$ & & & & - & & & - \\
\hline EGFR-TKI & $78(20.4)$ & $72(92.3)$ & $6(7.7)$ & & $78(100)$ & 0 & \\
\hline Chemotherapy & $305(79.6)$ & $222(72.8)$ & $83(27.2)$ & & 89 (29.2) & $216(70.8)$ & \\
\hline Tumor progression $(n=397)$ & & & & .061 & & & .072 \\
\hline Yes & $139(35.0)$ & $115(82.7)$ & $24(17.3)$ & & $70(50.4)$ & $69(49.6)$ & \\
\hline No & $258(65.0)$ & $192(74.4)$ & $66(25.6)$ & & $105(40.7)$ & $153(59.3)$ & \\
\hline Tumor related deaths $(n=397)$ & & & & .502 & & & $>.990$ \\
\hline Yes & $30(7.6)$ & $25(83.3)$ & $5(16.7)$ & & $13(43.3)$ & $17(56.7)$ & \\
\hline No & $367(92.4)$ & $282(76.8)$ & 85 (23.2) & & $162(44.1)$ & $205(55.9)$ & \\
\hline Specimen type & & & & & & & - \\
\hline Resections & $19(2.0)$ & $16(84.2)$ & $3(15.8)$ & - & $5(26.3)$ & $14(73.7)$ & \\
\hline Small biopsies & $890(98.0)$ & $682(76.6)$ & $208(23.4)$ & & $264(29.7)$ & $626(70.3)$ & \\
\hline Histological diagnosis & & & & & & & - \\
\hline AC/NSCLC, favor AC & $815(89.7)$ & $698(85.6)$ & $117(14.4)$ & - & $261(32.0)$ & $554(68.0)$ & \\
\hline NSCLC, NOS & $94(10.3)$ & 0 & $94(100)$ & & $8(8.5)$ & $86(91.5)$ & \\
\hline EGFR mutation type & & & & & & & - \\
\hline Exon 19 deletion & $146(16.1)$ & $133(91.1)$ & $13(8.9)$ & $>.990$ & - & - & \\
\hline Exon 21 L858R & $65(7.2)$ & 60 (92.3) & $5(7.7)$ & & & & \\
\hline Uncommon/dual & $58(6.4)$ & $49(84.5)$ & $9(15.5)$ & & & & \\
\hline None & $640(70.4)$ & $456(71.3)$ & $184(28.7)$ & & & & \\
\hline
\end{tabular}

TTF-1, thyroid transcription factor 1; EGFR, epidermal growth factor receptor; SD, standard deviation; TKI, tyrosine kinase inhibitor; AC, adenocarcinoma; NSCLC, non-small cell lung carcinoma; NOS, not otherwise specified.

common EGFR mutations (both exon 19 deletion and exon 21 L858R combined at 28\%) than with uncommon/dual mutations (7\%). The overall NPV of TTF-1 for EGFR mutations was $87 \%$, was increased to $91 \%$ in males and for common EGFR mutations, and was highest for uncommon/dual mutations (96\%).

We also calculated the diagnostic odds ratio (OR) within the entire cohort and within distinct clinicopathological subsets. OR denotes the ratio of the odds of TTF-1 being positive in an EGFR mutant tumor relative to the odds of TTF-1 being positive in an EGFR wild type tumor in this study and is independent of prevalence of EGFR mutations in the group under study. Overall, we found that TTF-1 was 3.6 times more likely to be positive in EGFR mutant tumors compared to EGFR wild type (OR, 3.61), with strong statistical strength $(\mathrm{p}<.001)$. This positive correlation between TTF-1 and EGFR mutation status remained sig- nificant within all individual clinicopathological subsets except when only considering uncommon/dual EGFR mutations (Table 2, Fig. 2). The highest OR was observed in non-smokers (OR, 6.53) and among those with common EGFR mutations (OR, 4.09), with no significant difference in OR between the two common mutations, exon 19 deletion (OR, 3.59; $95 \%$ confidence interval [CI], 2.06 to 6.79; $\mathrm{p}<.001)$ and exon $21 \mathrm{~L} 858 \mathrm{R}(\mathrm{OR}$, 3.88 ; $95 \%$ CI, 1.69 to $11.20 ; \mathrm{p}=.001$ ).

\section{Histopathological subtyping based on TTF-1 and EGFR mutation status}

\section{TTF-1_positive EGFR-positive cases ( $n=242)$}

Most of these cases were classified as AC $(n=235)$. Other rare subtypes included one case each of invasive mucinous AC and 
Table 2. Correlation between TTF-1 immunohistochemistry and EGFR mutations

\begin{tabular}{|c|c|c|c|c|c|c|c|c|}
\hline \multirow{2}{*}{$\begin{array}{l}\text { TTF- } 1 \text { IHC/ EGFR } \\
\text { mutation status }\end{array}$} & \multicolumn{2}{|c|}{ No. $(\%)$} & \multirow{2}{*}{ Sensitivity (\%) } & \multirow{2}{*}{ Specificity (\%) } & \multirow{2}{*}{ PPV (\%) } & \multirow{2}{*}{ NPV (\%) } & \multirow{2}{*}{$\begin{array}{l}\text { Odds ratio } \\
(95 \% \mathrm{Cl})\end{array}$} & \multirow{2}{*}{$p$-value } \\
\hline & Mutation detected & No mutation detected & & & & & & \\
\hline \multicolumn{3}{|l|}{ Total } & & & & & & $<.001$ \\
\hline Positive & $242(90.0)$ & $456(71.2)$ & 90 & 29 & 35 & 87 & $3.61(2.38-5.68)$ & \\
\hline Negative & $27(10.0)$ & $184(28.8)$ & & & & & & \\
\hline \multicolumn{3}{|l|}{ Females } & & & & & & $<.001$ \\
\hline Positive & 108 (89.3) & $104(72.2)$ & 89 & 28 & 51 & 75 & $3.19(1.66-6.53)$ & \\
\hline Negative & $13(10.7)$ & $40(27.8)$ & & & & & & \\
\hline \multicolumn{3}{|l|}{ Males } & & & & & & $<.001$ \\
\hline Positive & $134(90.5)$ & $352(71.0)$ & 91 & 29 & 28 & 91 & $3.91(2.26-7.31)$ & \\
\hline Negative & $14(9.5)$ & $144(29.0)$ & & & & & & \\
\hline \multicolumn{3}{|l|}{ Non-smokers } & & & & & & $<.001$ \\
\hline Positive & $98(95.1)$ & $57(75.0)$ & 95 & 25 & 63 & 79 & $6.53(2.33-18.43)$ & \\
\hline Negative & $5(4.9)$ & $19(25.0)$ & & & & & & \\
\hline \multicolumn{3}{|l|}{ Smokers } & & & & & & .045 \\
\hline Positive & $40(77.0)$ & $82(62.6)$ & 77 & 37 & 33 & 80 & $1.99(0.95-4.16)$ & \\
\hline Negative & $12(23.0)$ & $49(37.4)$ & & & & & & \\
\hline \multicolumn{2}{|l|}{ Common EGFR mutations } & & & & & & & $<.001$ \\
\hline Positive & $193(91.5)$ & $505(72.3)$ & 91 & 28 & 28 & 91 & $4.09(2.46-6.83)$ & \\
\hline Negative & $18(8.5)$ & $193(27.7)$ & & & & & & \\
\hline \multicolumn{3}{|c|}{ Uncommon/dual EGFR mutations } & & & & & & .098 \\
\hline Positive & $49(84.5)$ & $649(76.3)$ & 84 & 24 & 7 & 96 & $1.69(0.82-3.51)$ & \\
\hline Negative & $9(15.5)$ & $202(23.7)$ & & & & & & \\
\hline
\end{tabular}

TTF-1, thyroid transcription factor 1; EGFR, epidermal growth factor receptor; $I \mathrm{HC}$, immunohistochemistry; PPV, positive predictive value; NPV, negative predictive value; $\mathrm{Cl}$, confidence interval.

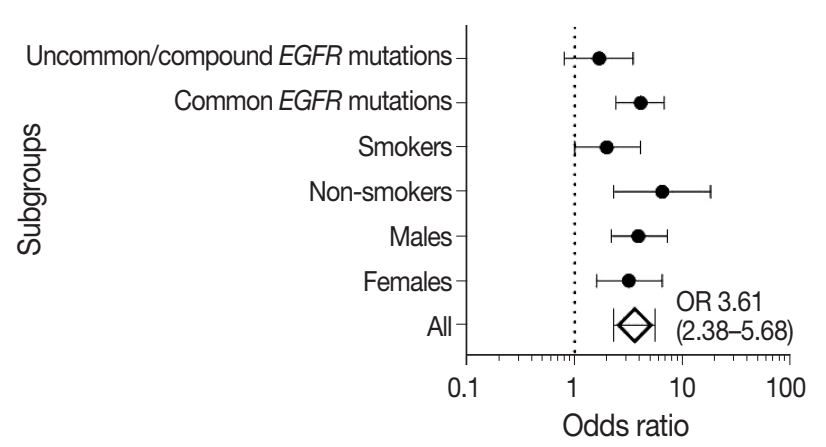

Fig. 2. Forest plot for correlation between thyroid transcription factor 1 and epidermal growth factor receptor (EGFR) mutation status in clinicopathological subgroups. Subgroup-specific odds ratios (ORs) are denoted by black dots (line width corresponding to 95\% confidence intervals). Combined OR estimate for all patients is represented by a black diamond, where the width corresponds to 95\% confidence intervals.

sarcomatoid carcinoma and five cases of adenosquamous carcinoma. The spectrum of EGFR mutations is shown in Fig. 3.

\section{TTF-1_negative EGFR-positive cases $(n=27)$}

Three cases were classified as invasive mucinous AC and one case as large cell carcinoma. Among the remaining cases, 16 cases classified as AC. EGFR mutations detected in these cases are shown in Fig. 3. Among dual mutations, five were E20 T790M in combination with other activating mutations [exon 19 deletion (4) and exon 18 G719X (1)] and two were combination of exon 19 deletion with exon 21 L858R.

\section{TTF-1_positive EGFR-negative cases $(n=456)$}

The majority of this group showed AC $(n=435)$ with predominant patterns, as shown in Fig. 3. The following subtypes were identified: three cases of sarcomatoid carcinoma, four cases of invasive mucinous AC, and six cases of adenosquamous carcinoma. A few rare variants were noted (signet cell morphology in six, cribriform architecture in one, and hepatoid in one case).

\section{TTF-1-negative EGFR-negative cases $(n=184)$}

This group showed AC in 88 cases (Fig. 3), invasive mucinous $\mathrm{AC}$ in five cases, and adenosquamous carcinoma in three cases. One case showed AC with signet ring cell differentiation. A total of 87 cases with solid architecture and without evidence of glandular differentiation were classified as NSCLC, NOS.

\section{Treatment details and survival analysis}

Treatment details were available for 383 patients, where 305 patients received platinum-based chemotherapy and 78 patients 

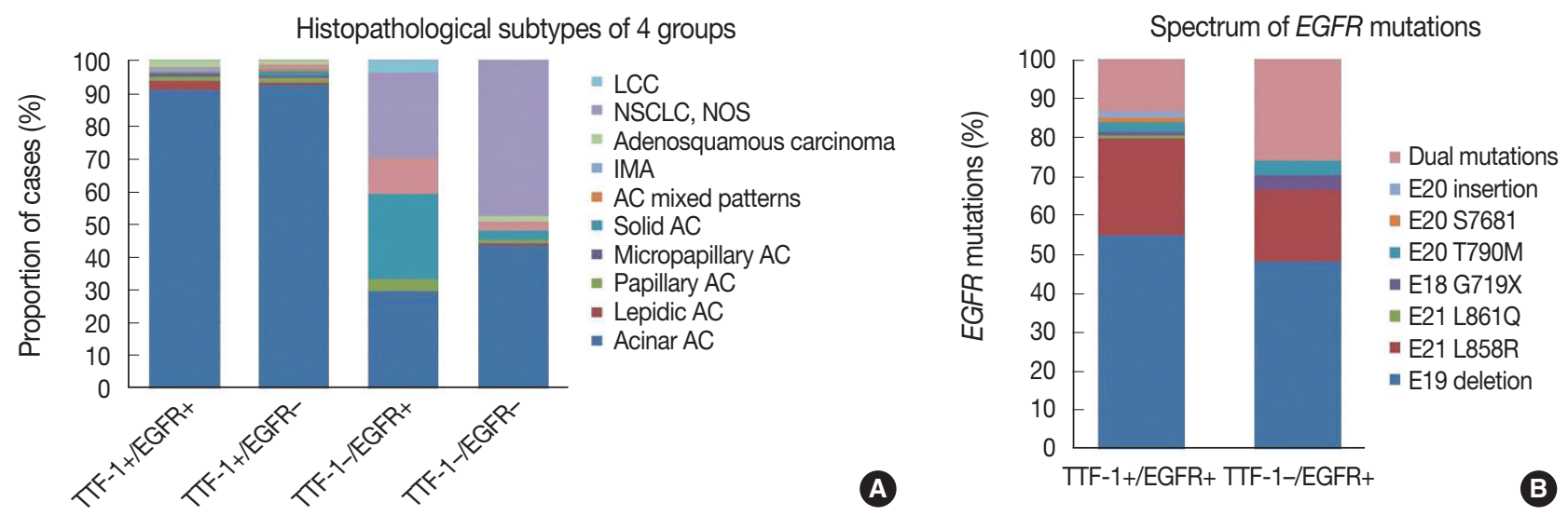

Fig. 3. Bar diagram showing histopathological subtyping of four groups based on thyroid transcription factor 1 (TTF-1) and epidermal growth factor receptor (EGFR) mutation status (A) and spectrum of EGFR mutations (B) in these groups. AC, adenocarcinoma; IMA, invasive mucinous adenocarcinoma; LCC, large cell carcinoma; NSCLC, non small cell lung carcinoma; NOS, not otherwise specified.

Table 3. Response to treatment with first-line EGFR-TKI treatment versus chemotherapy in the four patient groups

\begin{tabular}{lcccc}
\hline Parameter & TFF-1(+)/EGFR mutant & TTF-1(+)/EGFR wild type & TFF-1(-)/EGFR mutant & TFF-1(-)/EGFR wild type \\
\hline Treatment, $n$ (\%) & 114 & 83 & 14 & 38 \\
1st line EGFR-TKI & $49(43.0)$ & 0 & $4(28.6)$ & 0 \\
1st line CT & $65(57.0)$ & $83(100)$ & $10(71.4)$ & $38(100)$ \\
First-line TKI, $n$ (\%) & 49 & 0 & 4 & 0 \\
Responders & $35(71.4)$ & 0 & $3(75.0)$ & 0 \\
Non-responders & $14(28.6)$ & & $1(25.0)$ & 0 \\
First-line CT, $n$ (\%) & 65 & 83 & 10 & 38 \\
Responders & $30(46.2)$ & $29(34.9)$ & $3(30.0)$ & $11(28.9)$ \\
Non-responders & $35(53.8)$ & $54(65.1)$ & $7(70.0)$ & $27(71.9)$ \\
Odds ratio (TKI vs. CT) & 2.9 & 1 & 6.9 & 1 \\
95\% Cl & $1.32-6.42$ & - & $0.50-97.75$ & - \\
p-value & .008 & -148 & - \\
Median duration of follow-up (wk) & 23.2 & 20.4 & 22.1 & 22.6 \\
1-Year OS rate (\%) & 90.6 & 86.7 & 87.5 & 86.20 \\
Median PFS (mo) & 10.2 & 8.6 & 15.9 & 11.8 \\
HR for PFS & 1 & 1.07 & 0.83 & 0.92 \\
95\% Cl & & $0.74-1.56$ & $0.38-1.81$ & $0.53-1.58$ \\
p-value & & .694 & .631 & .754 \\
\hline
\end{tabular}

EGFR, epidermal growth factor receptor; TKI, tyrosine kinase inhibitor; TTF-1, thyroid transcription factor 1; CT, chemotherapy; OS, overall survival; PFS, progression-free survival; HR, hazard ratio; $\mathrm{Cl}$, confidence interval.

received EGFR-TKI therapy as first-line treatment. A total of 29.2\% of patients who received chemotherapy had EGFR mutated NSCLC, where treatment decisions were made before availability of EGFR mutation status results. Response to firstline therapy as per the Response Evaluation Criteria in Solid Tumors (RECIST) was available for 249 patients. Irrespective of TTF-1 and EGFR status, EGFR-TKI treatment resulted in superior response rates $(71.7 \%, 38 / 53)$ compared to chemotherapy $(37.2 \%, 73 / 196)(\mathrm{OR}, 3.47$; 95\% CI, 1.84 to 6.53 ; $0.48, \mathrm{p}<.001)$. For treatment response and survival analysis, patients were stratified into four groups based on TTF- 1 expression and EGFR mutations: TTF-1+/EGFR+, TTF-1+/EGFR-,
TTF-1-/EGFR+, and TTF-1-/EGFR-. Response to treatment in these four groups is depicted in Table 3. Among EGFR wild type cases, patients receiving chemotherapy and harboring TTF1-positive tumors responded better than patients with TTF-1negative tumors. However, this difference did not reach statistical significance (OR, 1.32 ; $95 \% \mathrm{CI}, 0.57$ to 3.03 ; $\mathrm{p}=.542$ ).

Survival data was available for 397 patients. The median duration of follow-up was 5.3 months. Overall, $35.01 \%$ of the patients experienced tumor progression during the course of therapy, and $7.6 \%$ of patients died. Patients with EGFR mutation and TTF-1 expression showed the best overall one-year survival rate $(90.6 \%)$, while those who were double negative showed the 
shortest (86.2\%), although these differences did not reach statistical significance. Notably, the OS rates among patients with EGFR wild type tumors positive for TTF-1 (86.7\%) and negative for TTF-1 (86.2\%) were very similar. On the other hand, progression-free survival (PFS) was more homogenous and the PFS durations and hazard ratios for progression were comparable among the four groups.

\section{DISCUSSION}

Many observational studies over the years have reported strong positive correlation between TTF-1 expression and EGFR mutation status [17-22]. From a meta-analysis of 9,764 patients, Kim et al. [23] reported an OR of 5 between TTF-1 and EGFR mutations, and the OR was similar among European $(\mathrm{OR}, 4)$ and Asian (OR, 4) cohorts. The only previous study from India on a smaller South Indian cohort of 85 patients with advanced NSCLC reported an OR of 15 between TTF- 1 and EGFR mutations [19]. In our single-institutional North Indian cohort of 909 patients, we found a similar strong correlation between TTF-1 and EGFR mutations (OR, 3.61) comparable to the results observed previously [17-23].

While EGFR mutations are more frequent in females and nonsmokers $[13,20,24]$, TTF-1 expression in NSCLC has shown inconsistent correlation with clinical parameters [21,25]. In the present study comprising predominantly of advanced stage nonsquamous NSCLC diagnosed on small biopsies, TTF-1 expression was seen in approximately $77 \%$ of cases and was significantly more common among non-smokers but lacked association with age or sex, while EGFR mutations were detected in about $30 \%$ of all cases and significantly more frequent among females, non-smokers, and at early clinical stages. The frequency of $E G F R$ mutation is similar to those reported in the Indian literature [13].

The correlation between TTF-1 and EGFR mutations in the present study remained significant irrespective of sex or smoking habit, indicating that the positive association is not merely due to increased prevalence of EGFR mutations in some demographic groups. Kim et al. [23] reported higher OR among females (OR, 4.87) compared to males (OR, 3.34), while Zhao et al. [21] reported lack of significant association between TTF-1 and $E G F R$ mutations in males. In the present study, we observed comparable results between males and females, with ORs slightly higher among males (OR, 3.91) than females (OR, 3.19). Similarly, although non-smokers showed the highest ORs (6.53), there was significant positive correlation of TTF-1-EGFR among smokers as well, albeit with a lower OR of 1.99 , as has been observed pre- viously [21]. The degree of expression of TTF-1 (i.e., in $>50 \%$ of tumor cells or in 10\%-50\% of tumor cells) also does not appear to show significant difference in positive association with EGFR mutations, especially the exon 21 mutation [25]. In our study, we used a cutoff of $>5 \%$ tumor nuclei staining for positivity.

Morphological analysis of the TTF-1-negative cases harboring EGFR mutations showed histological evidence of $\mathrm{AC}$ in $70.4 \%$ of cases. On further subtyping of these cases, the association of EGFR mutations with predominant subtyping of the cases with TTF-1 negativity was akin to the published literature [26]. Acinar predominant AC showed higher propensity for EGFR mutations. A few cases of mucinous AC, which were mostly TTF-1 negative, also were positive for $E G F R$ mutations.

The strength of correlation between TTF- 1 and EGFR appears to vary according to type of mutation. While initial studies found significant association with exon 21 mutations and not with exon 19 deletion [21,25], others have found significant association of TTF-1 expression with both of these common mutations [23, $27,28]$. We found strong association with both types of TKI-sensitive activating mutations, with an OR slightly higher for exon 19 deletion (OR, 4.63) than for exon 21 L858R (OR, 3.16). Interestingly, as has been observed in one previous study [27], we did not find significant correlation of TTF-1 with uncommon/ dual mutations (OR, 1.69; $\mathrm{p}>.05$ ).

The reported literature also states that more than $90 \%$ of EGFR mutated lung ACs are positive for TTF-1 [20-22,29]. In the present study, TTF-1 positivity showed $90 \%$ sensitivity for EGFR mutations, and the sensitivity was higher among males and non-smokers. Considering that TTF-1 positivity is not specific for $E G F R$ mutations, with only $34.6 \%$ of all TTF-1 positive tumors harboring EGFR mutations, the NPV rather than the PPV becomes the important criterion in our assessment of the value of TTF-1 IHC as a screening tool for EGFR mutations. We found that TTF-1 IHC shows NPV of $87 \%$ for EGFR mutations, comparable to the NPVs of $88 \%-97 \%$ reported in published cohorts [17,19,23,30-32]. NPV varies with prevalence of EGFR mutations and sensitivity of TTF-1 IHC [33] and is likely to be higher in populations with lower prevalence of $E G F R$ mutations, e.g., Western cohorts [30,31], and with the use of more sensitive TTF-1 IHC assays such as the SPT24 antibody clone (vs. 8G7G3/1) [9]. In line with these results, the highest NPVs in the present study were observed among males and among patients with uncommon/dual mutations.

EGFR mutation-specific antibodies have high specificity (94\%-100\%) and sensitivity (60\%-100\%) with high PPV and NPV for EGFR mutations compared to TTF-1 IHC. These an- 
tibodies have shown high concordance rate compared to the gold standard molecular techniques [34]. However, its interpretation is highly variable due to differences in criteria for positivity, and its practical application is limited as these antibodies are meant to detect the two most common mutations (exon 19 deletion and exon 21 L858R), with significant numbers of false positive and false negative cases [34]. A molecular-based assay is needed to confirm EGFR mutation status, and screening of EGFR mutations by IHC seems not to be applicable [35]. TTF-1 is a diagnostic marker that can be used as a screening tool for EGFR mutations, but it cannot be replaced by EGFR mutation-specific antibodies.

The high NPV of TTF-1 for EGFR mutations has led some to suggest that patients with negative TTF-1 can be started on chemotherapy without MT in time- and resource-constrained settings [31]. We found, however, that approximately $13 \%$ of TTF-1-negative tumors harbored EGFR mutations, $50 \%$ of which were TKI-sensitive exon 19 deletion and exon 21 L858R mutations. Thus, until greater clarity is achieved on the tumor response rates of TTF-1-negative/EGFR mutant tumors for EGFR-TKI vs. chemotherapy, excluding patients for MT based on TTF-1 negativity should be discouraged.

Activating EGFR mutations are established as predictive biomarkers for TKI responsiveness in NSCLC and are known to confer a favorable prognostic effect on recurrence-free survival and OS irrespective of treatment arms [36,37]. Similarly, TTF1 expression has been reported as a favorable prognostic biomarker in NSCLCs [11,12,38-40]. Transcriptional activation of TTF-1 has been shown to be necessary for EGFR downstream signaling in EGFR mutant tumors [41], and TTF-1 has been suggested to correlate with EGFR oncogene addiction in these tumors [28]. Tumors showing dual positivity for EGFR mutations and TTF-1 IHC have shown the best outcomes, while those negative for both carried the worst outcomes $[12,21,28]$. TTF-1 positive/EGFR wild type tumors and TTF-1 negative/ $E G F R$ mutant tumors show intermediate outcomes, with the former showing longer progression-free and OS than the latter in some studies $[12,21,28]$. In the present study, limited by availability of patient follow-up data, one-year OS was highest among TTF-1-positive/EGFR mutant cases and lowest for dual negative cases. TTF-1 positivity did not appear to alter the oneyear OS or PFS among EGFR wild type tumors, as shown in previous studies $[12,21,28]$. The improved survival of TTF-1-positive/ $E G F R$ wild type cases can be attributed in part to the presence of other prognostically favorable alterations such as ALK or BRAF [42]; however, these alterations were not analyzed in our study.
TTF-1 positivity has been correlated with better response rates to chemotherapy [28,39,43] and EGFR-TKI [39], including some EGFR wild type tumors [28,38]. With regard to chemotherapy, we did observe slightly better response rates in TTF1-positive/EGFR wild type cases compared to TTF-1-negative/ EGFR wild type cases. However, with regard to EGFR-TKI, we found no differences in response rates between TTF-1-positive and TTF-1-negative EGFR mutant cases. None of our EGFR wild type cases received TKI treatment; hence, we could not analyze TKI response rates based on TTF-1 expression status. Nevertheless, our observations lack statistical significance, and observational trials including more patients are necessary to confirm or refute previous observations.

\section{Conclusion}

In agreement with previous studies, we found a strong positive correlation between TTF-1 expression and common EGFR mutations (exon 19 deletion and exon 21 L858R) in advanced lung AC. The strength of correlation was highest among nonsmokers and lowest among those with uncommon/dual mutations. Despite a high NPV of TTF-1 IHC for EGFR mutations in the present study and previous studies, a better understanding of TTF-1-mediated modulation of treatment responses and its underlying biological mechanisms in EGFR mutant ACs is necessary before considering implementation of TTF-1 IHC as a surrogate for $E G F R$ mutations.

\section{Ethics Statement}

Approval from the Institute Ethics Committee (IECPG-480/29.08.2016), All India Institute of Medical Sciences, was obtained to conduct this retrospective study on archival patient samples. Thus, the informed consent was waived due to the retrospective nature of this study.

\section{Availability of Data and Material}

The datasets generated or analyzed during the study are available from the corresponding author on reasonable request.

\section{Code Availability}

Not applicable.

\section{ORCID}

Tripti Nakra

Varsha Singh

Aruna Nambirajan

Prabhat Singh Malik

Anant Mohan

Deepali Jain

https://orcid.org/0000-0001-9907-7531
https://orcid.org/0000-0002-2761-7102
https://orcid.org/0000-0001-7589-537X
https://orcid.org/0000-0003-0205-8559
https://orcid.org/0000-0002-2383-9437
https://orcid.org/0000-0001-5315-9814

Author Contributions

Conceptualization: DJ. Data curation: TN, VS, AN, PSM, AM, DJ. Formal analysis: TN, VS, AN, DJ. Methodology: TN, VS, AN, DJ. Writing_original 
draft: TN. Writing—review \& editing: TN, VS, AN, PSM, AM, DJ. Approval of final manuscript: all authors.

\section{Conflicts of Interest}

D.J., a contributing editor of the Journal of Pathology and Translational Medicine, was not involved in the editorial evaluation or decision to publish this article. All remaining authors have declared no conflicts of interest.

\section{Funding Statement}

This work was supported by Department of Biotechnology (grant number N-1611) and The Lady Tata Memorial Trust (grant number N-1590).

\section{References}

1. Yatabe Y, Mitsudomi T, Takahashi T. TTF-1 expression in pulmonary adenocarcinomas. Am J Surg Pathol 2002; 26: 767-73.

2. Lau SK, Luthringer DJ, Eisen RN. Thyroid transcription factor-1: a review. Appl Immunohistochem Mol Morphol 2002; 10: 97-102.

3. Walia R, Jain D, Madan K, et al. p40 \& thyroid transcription factor-1 immunohistochemistry: a useful panel to characterize nonsmall cell lung carcinoma-not otherwise specified (NSCLC-NOS) category. Indian J Med Res 2017; 146: 42-8.

4. Yatabe Y, Dacic S, Borczuk AC, et al. Best practices recommendations for diagnostic immunohistochemistry in lung cancer. J Thorac Oncol 2019; 14: 377-407.

5. Malik PS, Sharma MC, Mohanti BK, et al. Clinico-pathological profile of lung cancer at AIIMS: a changing paradigm in India. Asian Pac J Cancer Prev 2013; 14: 489-94.

6. Noronha V, Pinninti R, Patil VM, Joshi A, Prabhash K. Lung cancer in the Indian subcontinent. South Asian J Cancer 2016; 5: 95-103.

7. Lindeman NI, Cagle PT, Aisner DL, et al. Updated molecular testing guideline for the selection of lung cancer patients for treatment with targeted tyrosine kinase inhibitors: guideline from the College of American Pathologists, the International Association for the study of lung cancer, and the Association for Molecular Pathology. J Thorac Oncol 2018; 13: 323-58.

8. Jain D, Iqbal S, Walia R, et al. Evaluation of epidermal growth factor receptor mutations based on mutation specific immunohistochemistry in non-small cell lung cancer: a preliminary study. Indian J Med Res 2016; 143: 308-14.

9. Inamura K. Update on immunohistochemistry for the diagnosis of lung cancer. Cancers (Basel) 2018; 10: 72.

10. Suda K, Mitsudomi T. Role of EGFR mutations in lung cancers: prognosis and tumor chemosensitivity. Arch Toxicol 2015; 89: 122740.

11. Berghmans T, Paesmans M, Mascaux C, et al. Thyroid transcription factor 1: a new prognostic factor in lung cancer: a meta-analysis. Ann Oncol 2006; 17: 1673-6.

12. Chung KP, Huang YT, Chang YL, et al. Clinical significance of thyroid transcription factor- 1 in advanced lung adenocarcinoma under epidermal growth factor receptor tyrosine kinase inhibitor treatment. Chest 2012; 141: 420-8.

13. Nakra T, Mehta A, Bal A, et al. Epidermal growth factor receptor mutation status in pulmonary adenocarcinoma: multi-institutional data discussion at national conference of "Lung Cancer Management in Indian context". Curr Probl Cancer 2020; 44: 100561.

14. Singh V, Guleria P, Malik PS, et al. Epidermal growth factor receptor (EGFR), KRAS, and BRAF mutations in lung adenocarcinomas: a study from India. Curr Probl Cancer 2019; 43: 391-401.
15. Singh V, Nambirajan A, Malik PS, et al. Spectrum of uncommon and compound epidermal growth factor receptor mutations in nonsmall-cell lung carcinomas with treatment response and outcome analysis: a study from India. Lung Cancer 2020; 149: 53-60.

16. Travis WD, Brambilla E, Burke AP, Marx A, Nicholson AG. WHO classification of tumours of the lung, pleura, thymus and heart. 4th ed. Lyon: IARC Press, 2015.

17. Sheffield BS, Bosdet IE, Ali RH, et al. Relationship of thyroid transcription factor 1 to EGFR status in non-small-cell lung cancer. Curr Oncol 2014; 21: 305-8.

18. Carneiro JG, Couto PG, Bastos-Rodrigues L, et al. Spectrum of somatic EGFR, KRAS, BRAF, PTEN mutations and TTF-1 expression in Brazilian lung cancer patients. Genet Res (Camb) 2014; 96: e002.

19. Udupa KS, Rajendranath R, Sagar TG, Sundersingh S, Joseph T. Dual surrogate markers for rapid prediction of epidermal growth factor receptor mutation status in advanced adenocarcinoma of the lung: a novel approach in resource-limited setting. Indian J Cancer 2015; 52: 266-8

20. Sun PL, Seol H, Lee HJ, et al. High incidence of EGFR mutations in Korean men smokers with no intratumoral heterogeneity of lung adenocarcinomas: correlation with histologic subtypes, EGFR/TTF1 expressions, and clinical features. J Thorac Oncol 2012; 7: 323-30.

21. Zhao Q, Xu S, Liu J, et al. Thyroid transcription factor-1 expression is significantly associated with mutations in exon 21 of the epidermal growth factor receptor gene in Chinese patients with lung adenocarcinoma. Onco Targets Ther 2015; 8: 2469-78.

22. Gahr S, Stoehr R, Geissinger E, et al. EGFR mutational status in a large series of Caucasian European NSCLC patients: data from daily practice. Br J Cancer 2013; 109: 1821-8.

23. Kim HS, Kim JH, Han B, Choi DR. Correlation of thyroid transcription factor-1 expression with EGFR mutations in non-smallcell lung cancer: a meta-analysis. Medicina (Kaunas) 2019; 55: 41.

24. Travis WD, Noguchi M, Yatabe Y, et al. Adenocarcinoma. In: Travis WD, Brambilla E, Burke AP, Marx A, Nicholson AG. WHO classification of tumours of the lung, pleura, thymus and heart. 4th ed. Lyon: IARC Press, 2015; 46-51.

25. Shanzhi W, Yiping H, Ling H, Jianming Z, Qiang L. The relationship between TTF-1 expression and EGFR mutations in lung adenocarcinomas. PLoS One 2014; 9: e95479.

26. Dong YJ, Cai YR, Zhou LJ, et al. Association between the histological subtype of lung adenocarcinoma, EGFR/KRAS mutation status and the $A L K$ rearrangement according to the novel IASLC/ATS/ ERS classification. Oncol Lett 2016; 11: 2552-8.

27. Wei WE, Mao NQ, Ning SF, et al. An analysis of EGFR mutations among 1506 cases of non-small cell lung xancer patients in Guangxi, China. PLoS One 2016; 11: e0168795.

28. Park JY, Jang SH, Kim HI, et al. Thyroid transcription factor-1 as a prognostic indicator for stage IV lung adenocarcinoma with and without EGFR-sensitizing mutations. BMC Cancer 2019; 19: 574.

29. Vallee A, Sagan C, Le Loupp AG, Bach K, Dejoie T, Denis MG. Detection of EGFR gene mutations in non-small cell lung cancer: lessons from a single-institution routine analysis of 1,403 tumor samples. Int J Oncol 2013; 43: 1045-51.

30. Somaiah N, Fidler MJ, Garrett-Mayer E, et al. Epidermal growth factor receptor (EGFR) mutations are exceptionally rare in thyroid transcription factor (TTF-1)-negative adenocarcinomas of the lung. Oncoscience 2014; 1: 522-8.

31. Vincenten J, Smit EF, Vos W, et al. Negative NKX2-1 (TTF-1) as 
temporary surrogate marker for treatment selection during EGFRmutation analysis in patients with non-small-cell lung cancer. J Thorac Oncol 2012; 7: 1522-7.

32. Jie L, Li XY, Zhao YQ, et al. Genotype-phenotype correlation in Chinese patients with pulmonary mixed type adenocarcinoma: relationship between histologic subtypes, TITF-1/SP-A expressions and EGFR mutations. Pathol Res Pract 2014; 210: 176-81.

33. Lange K, Brunner E. Analysis of predictive values based on individual risk factors in multi-modality trials. Diagnostics (Basel) 2013; 3 : 192-209.

34. Gaber R, Goldmann T. Mini review: immunohistochemistry using EGFR-mutant specific antibodies in non-small cell lung carcinoma: accuracy and reliability. J Cancer Treatment Diagn 2018; 2: 17-23.

35. Jiang G, Fan C, Zhang X, et al. Ascertaining an appropriate diagnostic algorithm using EGFR mutation-specific antibodies to detect EGFR status in non-small-cell lung cancer. PLoS One 2013; 8: e59183.

36. Gazdar AF. Activating and resistance mutations of EGFR in nonsmall-cell lung cancer: role in clinical response to EGFR tyrosine kinase inhibitors. Oncogene 2009; 28 Suppl 1: S24-31.

37. Nan X, Xie C, Yu X, Liu J. EGFR TKI as first-line treatment for patients with advanced EGFR mutation-positive non-small-cell lung cancer. Oncotarget 2017; 8: 75712-26.
38. Nakahara Y, Hosomi Y, Saito M, et al. Predictive significance of thyroid transcription factor-1 expression in patients with non-squamous non-small cell lung cancer with wild-type epidermal growth factor receptor treated with erlotinib. Mol Clin Oncol 2016; 5: 14-8.

39. Sun JM, Han J, Ahn JS, Park K, Ahn MJ. Significance of thymidylate synthase and thyroid transcription factor 1 expression in patients with nonsquamous non-small cell lung cancer treated with pemetrexed-based chemotherapy. J Thorac Oncol 2011; 6: 1392-9.

40. Tang X, Kadara H, Behrens C, et al. Abnormalities of the TITF-1 lineage-specific oncogene in NSCLC: implications in lung cancer pathogenesis and prognosis. Clin Cancer Res 2011; 17: 2434-43.

41. Yamaguchi T, Yanagisawa K, Sugiyama R, et al. NKX2-1/TITF1/ TTF-1-induced ROR1 is required to sustain EGFR survival signaling in lung adenocarcinoma. Cancer Cell 2012; 21: 348-61.

42. Kret A, Clark C, Tennant S, Nicolson M, Kerr KM. Mutation analysis and association with TTF1 expression in lung non-squamous NSCLC. Lung Cancer 2015; 87(Suppl 1): S16.

43. Gronberg BH, Lund-Iversen M, Strom EH, Brustugun OT, Scott H. Associations between TS, TTF-1, FR-alpha, FPGS, and overall survival in patients with advanced non-small-cell lung cancer receiving pemetrexed plus carboplatin or gemcitabine plus carboplatin as first-line chemotherapy. J Thorac Oncol 2013; 8: 1255-64. 